\title{
A framework for feature based CAD/CAM integration
}

\author{
C.F. Zhu, N.N.Z. Gindy and Y. Yue \\ Department of Manufacturing Engineering and Operations Management \\ University of Nottingham, Nottingham NG7 2RD, UK \\ Tel +44-115-9514031, Fax +44-115-9514000 \\ Emailepxcfz@unicorn.nott.ac.uk
}

\begin{abstract}
This paper proposes a framework for a CAD/CAPP/CNC integration using the latest solid modelling and CNC development tools. The central concept for the integration is to use a generalised component data model throughout all the activities from design to manufacture. A feature-based component data model (FBCDM) is introduced. The creation and enhancement of FBCDM are also described.
\end{abstract}

\section{Keywords}

CAD/CAM integration, CAPP, component model, features, geometric reasoning

\section{INTRODUCTION}

The goal of integrating computer aided design and manufacturing (CAD and CAM) into computer integrated manufacturing (CIM) has been long desired. It is understood that it would be impossible to achieve real integration between CAD and CAM without automating computer aided process planning (CAPP). One of the problems encountered here is that it is difficult to interpret component design automatically by a computer for use in downstream applications in the production process (Anting and Zhang 1990, ElMaraghy 1993).

This paper proposes a framework for a CAD/CAPP/CNC integration using the latest solid modelling and computer numerical control (CNC) development tools. The central concept for the integration is to use a generalised form of component data throughout all the activities from design to manufacture. In the following sections, definitions for features including form features, design features and machining features are discussed, and a feature-based component data model (FBCDM) suitable for design, process planning and CNC programming is introduced. An intelligent feature based design system (IFBDS) is used to creates a generic FBCDM containing basic component information which is enriched through geometric reasoning. A feature based process planning system (FBPPS) extends and refines the FBCDM and automatically generates 
a process plan. The refined FBCDM is then directly used by a feature based numerical control programming system (FBNCPS) to generate the cutter path. A preliminary investigation of the framework has indicated a high potential of the methodology employed to achieve a fully integrated $\mathrm{CAD} / \mathrm{CAPP} / \mathrm{CNC}$ system.

\section{ARCHITECTURE OF THE CAD/CAPP/CNC INTEGRATION SYSTEM}

The architecture of the CAD/CAPP/CNC integration system is depicted in Figure 1. It consists of three subsystems: IFBDS, FBPPS and FBNCPS.

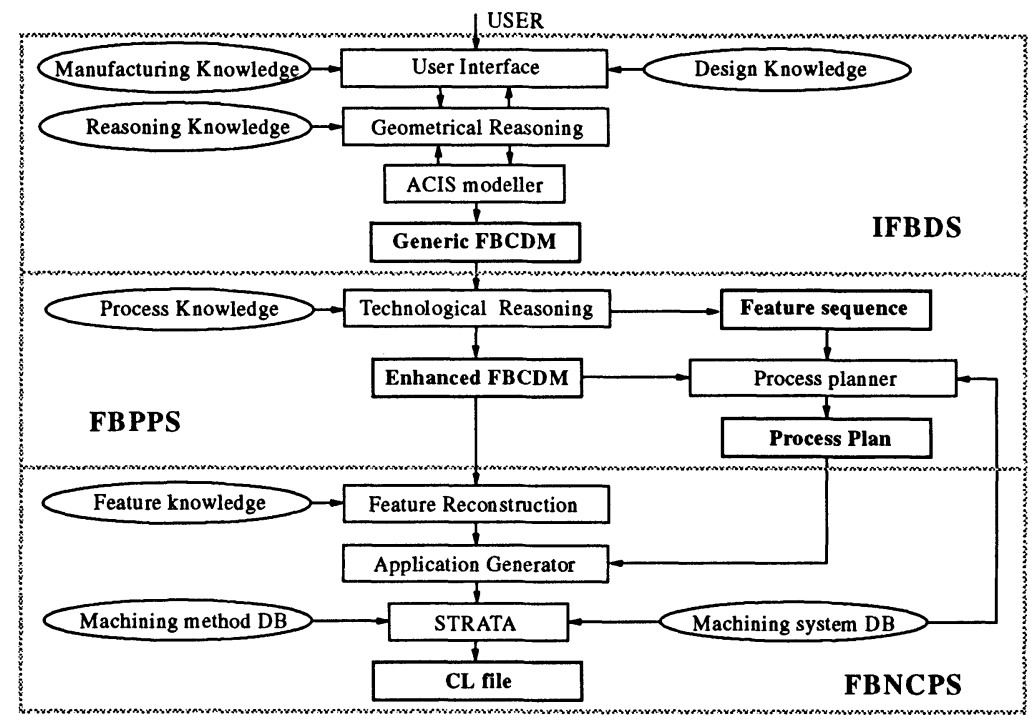

Figure 1 System architecture.

IFBDS uses ACIS (Spatial Technology Inc.1994) as its underlying modeller. It has a user interface with design and manufacturing knowledge, an inference engine with reasoning capabilities, and an attribute tagging facility. The output is a generic FBCDM. The user interface contains a user-system interaction window in which command menus are provided. It allows the user to make parametric designs using the feature library, or modify/edit existing designs. A design knowledge database contains the feature library, standards and other necessary information; and a manufacturing knowledge database stores information on raw materials, machines, tools and fixtures.

In front of ACIS, there is an inference engine, the core of IFBDS, that serves three purposes: finding information of constituent entities of features, checking feature validity and identifying feature interactions. These tasks are mainly performed through geometric reasoning during the design stage. 
FBPPS comprises a design interface and a process planner. The function of the design interface is to take the generic FBCDM as input, enhance it through technological reasoning and refines the interacting features so that they can be treated individually for machining. Feature refinement aspects include the feature geometry, technological constraints and potential technological solutions. The feature sequence is also determined.

Based on the enhanced FBCDM, the process planner automatically determines the technological solutions for each feature, taking into account its technological constraints and the processing system capabilities. FBPPS output is an enhanced FBCDM and a process plan for the component.

FBNCPS uses Strata development environment (Spatial Technology Inc. 1994). It consists of a system interface, an application program generator and Strata CNC code generator. The system interface takes the enhanced FBCDM as input, converts features into Strata geometric entities with manufacturing information and reconstructs some of the features if necessary.

Based on the enhanced FBCDM and process plan, the application program generator automatically creates program in Strata command format. Strata executes the application program and creates a CNC toolpath for each feature. The toolpaths may be grouped into operations, while operations may be clustered into setups and setups into sub-programs which are combined into a full CNC program for the component. FBNCPS output is a cutter location (CL) file.

The key issues of integration between CAD/CAPP/CNC are the creation and enhancement of FBCDM as described in Sections 3 and 4.

\section{FEATURE-BASED COMPONENT DATA MODEL}

Conventional geometric models are created from geometric primitive objects (e.g. cylinders, blocks) by Boolean operations or from lines and curves by other geometric construction techniques (e.g. sweeping profiles to get volumes, or fitting a skin to a series of cross sections arranged in the space). They have proved deficient for applications such as automated process planning. Feature based models which provide higher conceptual meaning of component characteristics are considered as a suitable communication medium between design and manufacturing.

\subsection{Feature definition}

Many definitions appear in the literature (Case and Gao, 1993) since features originate from various design, analysis and manufacturing activities, and are often associated with particular application domains. In essence, features represent the engineering meaning of the geometry of a component (Shah 1992). Form features, simply defined as portions of nominal geometry or recurring shapes, have been widely used.

Since features are often application-specific, they can be defined and classified from the viewpoint of different processes occurring in the product life-cycle (Pratt 1993, Young and Bell 1993). Design and manufacturing features are the two most common types: design features are geometry and specifications which fulfil certain functional requirements while manufacturing features are geometry that can be related to specific manufacturing methods. 
It can be seen from the above discussion that all application-specific features are related back to form features. Therefore design features can be defined as form features with technological constraints while manufacturing features as form features with technological solutions.

\subsection{Feature taxonomy}

While it is difficult to enumerate all features, it is useful to try to classify them. This research uses Gindy's (1989) feature taxonomy in which form features are treated as volumes enveloped by entry/exit and depth boundaries. A feature may consist of any number of real surfaces and a fixed number $(0-6)$ of imaginary surfaces. Each surface has a direction represented by a normal vector (NV). The NV of each imaginary surface of a feature is termed an external access direction (EAD) which represents a possible machining direction for the feature.

The features are divided into three categories: protrusion, depression and surface based on the number of their imaginary surfaces (i.e. EADs). These categories are further divided into nine classes based upon the feature boundary type (open or closed) and exit boundary status (through or not through). Each class of feature has a perimeter shape. This feature taxonomy is proving suitable to represent machined components for process planning and CNC programming. It is a hierarchical structure which can be implemented using object oriented techniques.

\subsection{A feature-based component data model}

FBCDM is used to represent the set of features included in component definition, their attributes and the relationships between features.

The feature list contains high-level information about each feature, such as its type, location, orientation, dimensions, accuracy and surface finish, some of which may be regarded as technological constraints for process planning and CNC programming.

From process planning point of view, there are a number of relationships between the features on a component, which are kept in the feature relationship list:

- Feature connectivity: Features with common EADs can be considered together for machining. The common EADs are called the potential approach directions (PADs) representing the possible machining directions for the features.

- Parent-child relationship: If features $\mathrm{A}$ and $\mathrm{B}$ are adjacent and $\mathrm{A}$ has an imaginary surface contained within the boundary of any real surface of $B$, then $A$ is the child feature of $B$ and $\mathrm{B}$ is the parent feature of the A. The parent-child relationship is useful in the decision-making (e.g. determining feature sequence).

- Compound feature: A compound feature is a set of primitive features treated as a single entity because they may perform a single function and/or need to be machined by the same procedure. In some cases, compound features have a parent-child relationship (e.g. a T-slot). Information of compound features can be used for determining setups.

- Tolerance relationship: When the designer specifies positional tolerances between features to guarantee some functional requirements, some tolerance relationships between the features are established. One feature can be considered the reference feature and the others tolerancing features. A tolerancing feature may have more than one reference feature and vice visa. The tolerance relationship is necessary for making setup and machining strategies. 
The ACIS data structure is extended to include the feature list and feature relationships. The advantage of FBCDM is that it provides sufficient and easily-accessible information for the applications while the model is kept in a generic and compact format.

\section{GENERATION AND ENHANCEMENT OF FBCDM}

A generic FBCDM is generated by the IFBDS through interactions between the user and the system. Geometric reasoning is performed where necessary; it minimises the demand on designers, and ensures that the model is valid and contains sufficient information for the downstream applications. The generic model is enhanced by technological reasoning to facilitate the FBPPS which then creates a process plan. The features in the enhanced FBCDM are reconstructed and used by the FBNCPS to generate a CL file.

\subsection{Geometric reasoning}

Based on component geometric information during the design stage, the tasks of geometric reasoning include the followings:

- Feature entity identification: Before a feature is added to the feature list, some of its constituent entities are identified and tagged with necessary information (e.g. tolerance and surface finish on a real face). The constituent entities include faces, edges and vertices among which faces are the most significant. The imaginary faces are identified using a predefined feature convention while other constituent entities are identified as necessary (e.g. when tolerance and surface finish attributes are to be tagged).

- Feature validity: This is a common problem in feature-oriented research. e.g. a designed slot may become a step due to feature interaction or manipulation. It is necessary to check that a feature is valid before it is added to the feature list. Thus ensuring the validity of the attributes tagged on the feature constituent entities.

- Parent-child feature identification: A valid feature's imaginary faces are examined against the real faces of other features. If an imaginary face lies within a real face of another feature, there is a parent-child relationship and this information is added to the model data.

\subsection{Technological reasoning}

The tasks of technological reasoning include feature sequencing, feature refinement and feature conversion based on both component geometric information and process knowledge.

\section{Feature sequencing}

Factors considered here include feature relationships such as parent-child relationships and tolerance relationships. The basic principles for feature sequence are:

- Reference features before other features;

- Parent features before child features if there are no other constraints;

- Main functional features before other features; 
- Large-sized features before small-sized features; and

- Orthogonal features before non-orthogonal features.

Feature sequence influences both operation sequence and features themselves which need to be refined based on the feature sequence. It may thus be necessary to change a feature sequence based on the feedback from feature refinement. Hence an iterative procedure is used.

\section{Feature refinement}

Because of feature interactions, it can be ambiguous to match design feature with manufacturing feature. This is solved by feature refinement, examples include:

- Feature splitting: Due to feature interaction, it is necessary to split a design feature into two or more manufacturing features to be machined separately (i.e. depending on the machining sequence). Figure 2a shows a design feature "hole" broken into hole1 and hole 2 by the slot which is machined first. If the length to diameter ratio $(V d)$ of the hole is too large for the hole to be drilled in one direction, it must be split into two holes for machining.

- Feature merging: On the other hand, it may be necessary to merge two or more design features into one manufacturing feature. For example, it is reasonable to treat hole 1 and hole 2 as a single hole for machining before the slot as illustrated in Figure $2 b$.

- Feature extension: A design feature may have to be extended owing to feature interactions and/or the allowance of raw material. Feature extension lengthens the feature volume as much as possible, e.g. the depth $h$ of the hole shown in Figure $2 \mathrm{c}$ can be extended to $h l$ if the hole is machined prior to the slot.

- Feature degeneration: Due to feature interactions and/or technological constraints, some EADs of a feature can not be used as feasible cutter access. Thus a design feature may become a manufacturing feature with fewer EADs by degeneration. Figure $2 \mathrm{~d}$ provides an example where a hole (through hole) is degenerated to a pocket (blind hole).

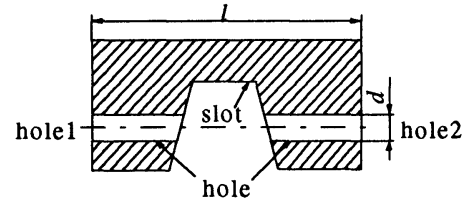

(a) Feature splitting.

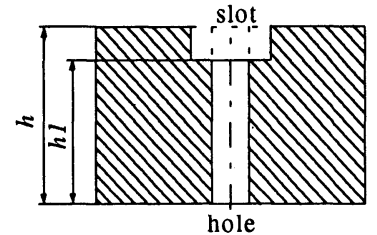

(c) Feature extension.

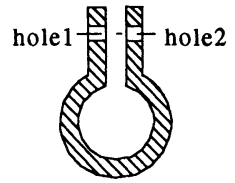

(b) Feature merging.

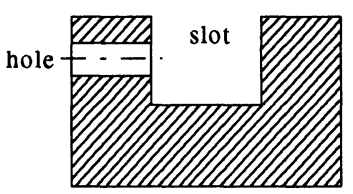

(d) Feature degenerating.

Figure 2 Feature refinement. 


\section{Feature conversion}

The design features after refinement are converted to manufacturing features by attaching manufacturing information called the technological solutions at the feature level (TSFs). A TSF is an ordered set of operations that satisfy the feature geometry and technological requirements (Gindy et al. 1993). Each operation is described as a form generation schema, i.e. a set of motions and tools for performing the operation, which facilitate machine tool and cutting tool selection in the process planning stage.

TSFs are stored in the manufacturing database according to feature transition diagrams (FTD) which represent the available processing methods for producing the feature. Based on the individual feature technological constraints, the technological solutions for producing a feature can be obtained by interrogating the manufacturing database.

\subsection{Feature reconstruction}

After feature sequencing, refinement and conversion, the generic FBCDM is enhanced. Due to the consistent use of manufacturing features defined in FBPPS and FBNCPS, most manufacturing features in the enhanced FBCDM can be used directly by FBNCPS. However, feature "hole" in Strata is different and has a special data structure. So it is necessary to reconstruct it from some manufacturing features in the enhanced FBCDM.

In Strata, feature "hole" is defined as a round pocket (blind hole) or round hole (through hole) machined by drilling (drilling, boring and reaming) operations, which can be identified using expert system rules. The rules for identifying Strata "hole" from the FBCDM can be written as follows:

IF a feature class is pocket or hole

AND its subclass is circular

AND its TSFs only include drilling, boring or reaming operations

THEN the feature is "hole"

Having been identified, feature "hole" can be recreated by Strata commands. Other features in the enhanced FBCDM are machined by milling and can be used directly by Strata.

\section{IMPLEMENTATION AND CONCLUSIONS}

The work discussed above is being implemented on a Sun SPARC station 10 with ACIS version 1.5.2 and Strata version 1.7.3. The major advantages of ACIS are its complete and open data structure and functionality which have made it possible to use the ACIS model as the core of the FBCDM. The ACIS attribute function has been used to tag attributes onto feature entities in generating a generic FBCDM. Strata is a next-generation manufacturing system based on ACIS models. It addresses operations planning and the automatic creation, validation, and verification of CNC toolpaths. The Strata command language provides a toolkit for developing CNC programming systems. These features of Strata have proved useful in the CAD/CAPP/CNC integration through the use of a generalised form of component data based on ACIS models.

The FBCDM is created by human-computer interaction in design stage. It contains sufficient information regarding the component which can be used by downstream applications such as 
process planning and $\mathrm{CNC}$ programming through automatic processing. This saves time and costs by avoiding repeat of input and reducing human errors. Furthermore, the current implementation of the framework has indicated a high potential of the methodology employed to achieve fully functional integration between $\mathrm{CAD}$ and CAM tasks.

\section{REFERENCES}

Alting, L. and Zhang, H.C. (1989) Computer-aided process planning: the state-of-the-art survey, Int. J. of Production Research, 27(4), 553-85.

Case, K. and Gao, J. 1993, Feature technology: an overview, Int. J. of Computer Integrated Manufacturing, 6(1 \& 2), 137-51.

EIMaraghy, H. (1993) Evaluation and future perspectives of CAPP, Annals of the CIRP, 42(2), 739-51.

Gindy, N.N.Z. (1989) A hierarchical structure for features, Int. J. of Production Research, 27(12), 2089-103.

Gindy, N.N.Z., Huang, X. and Ratchev, T.M. (1993) Feature based component model for computer aided process planning systems, Int. J. of Computer Integrated Manufacturing, 6(1\&2), 20-6.

Pratt, M.J. (1993) Applications of feature recognition in the product life-cycle, Int. J. of Computer Integrated Manufacturing, 6(1\&2), 13-9.

Shah, J.J. (1992) Features in design and manufacturing, Intelligent Design and Manufacturing, (ed. A. Kusiak), John Wiley \& Sons Inc.

Spatial Technology, Inc. (1994) ACIS Modeller Interface Guide.

Spatial Technology, Inc. (1994) Strata Command Reference.

Young, R.M. and Bell, R. (1993) Design by features: advantage and limitations in machine planning integration, Int. J. of Computer Integrated Manufacturing, 6(1 \& 2), 105-12.

\section{BIOGRAPHIES}

Cheng-Feng Zhu, B.Sc., M.Sc., is currently a Ph.D student at the Department of Manufacturing Engineering at the University of Nottingham, UK. He worked as a lecturer at Shanghai Jiao Tong University China until 1992. His major interests include CAD/CAM integration and Group Technology.

Nabil Gindy, B.Sc., M.Sc., Ph.D, is professor of Advanced Manufacturing Technology and leads the responsive manufacturing research group at the University of Nottingham, UK. His research interests include machining and tooling technology, feature-based CAD/CAM systems, generative process planning, integrated planning and scheduling systems, CNC part programming and the design and configuration of manufacturing systems.

Yong Yue, B.Sc., Ph.D, is currently a research assistant at the University of Nottingham, UK. He earned a Ph.D in 1994 from Heriot-Watt University, UK. He worked as a design and research engineer in Shenyang Aluminium and Magnesium Institute China from 1982 to 1990. His major interests include $\mathrm{CAD} / \mathrm{CAM}$ and integrated manufacturing. 